GANIT J. Bangladesh Math. Soc. (ISSN 1606-3694) 32 (2012) 61-73

\title{
NATURAL CONVECTION FLOW OF AN ELECTRICALLY CONDUCTING FLUID IN A RECTANGULAR CAVITY HAVING INTERNAL ENERGY SOURCES WITH LINEARLY HEATED BOTTOM WALL
}

\author{
M. Obayedullah and M. M. K. Chowdhury \\ Department of Mathematics \\ Bangladesh University of Engineering and Technology Dhaka-1000, Bangladesh
}

Email: obayed@math.buet.ac.bd

Received 20-05-2012 Accepted 03-11-2012

\begin{abstract}
Natural convection flow in a rectangular cavity containing internally heated and electrically conducting fluid has been investigated numerically. The bottom wall of the cavity is linearly heated whereas the top wall is well insulated. The left and right vertical walls are maintained at constant hot and cold temperature respectively. Results have been obtained with respect to Rayleigh numbers and Hartmann numbers. Flow and temperature fields for these cases have been studied. Average Nusselt numbers at hot, cold and linearly heated bottom wall have been calculated. It is found that the temperature, fluid flow and heat transfer strongly depend on internal and external Rayleigh numbers and Hartmann numbers.
\end{abstract}

Keywords: Natural convection, Heat generation, Rectangular cavity, Non-uniform heating.

\section{Introduction}

The influence of magnetic field on the natural convection flow of fluid is of great importance in the field of industry. Magnetohydrodynamic (MHD) principles are used in the design of heat exchangers, pumps and flow meters, in space vehicle propulsion and developing confinement schemes for controlled fusion. The extensive research studies using various numerical simulation reported by Oreper and Szekely[8], Ozoe and Maruo[9], Grandet et al.[2], Rudraiah et al.[10] and Chamkha[1]. ensure that several attempts have been made to acquire a basic understanding of natural convection flows and heat transfer characteristics in a rectangular enclosure having internal energy sources and electrically conducting fluid. However, in most studies, one vertical wall of the enclosure is cooled another one is heated while the remaining top and bottom wall are well insulated. Non-uniform temperature in the wall was not used in these studies.

The effect of surface tension on unsteady laminar natural convection flow of an electrically conducting fluid in a rectangular enclosure under an externally imposed magnetic field with internal heat generation has been investigated by Hossain et al.[3]. 
The top horizontal surface of the rectangular cavity is assumed to be free and the bottom one insulated, whereas the left vertical wall is cold and the right one is uniformly hot.

Sarris et al.[11] presented a numerical study of unsteady two dimensional natural convection of an electrically conducting fluid in a laterally and volumetrically heated square cavity under the influence of a magnetic field. Mehmet and Elif [6] studied the natural convection flow under a magnetic field in an inclined rectangular enclosure heated and cooled on adjacent walls.

Kandaswamy et al. [5] studied magnetohydrodynamic natural convection in an enclosure with partially active vertical walls. Nithyadevi et al.[7] investigated the natural convection flow under a magnetic field in a square cavity with partially active vertical walls having time periodic boundary condition. Kahveci et al.[4] studied magnetohydrodynamic natural convection flow and heat transfer in a laterally heated partitioned enclosure. Sathiyamoorty et al.[12] investigated the steady natural convection flow in a square cavity with linearly heated side walls. As per authorô knowledge the literature review revealed that no work has yet been done on the magnetohydrodynamic natural convection flow in a rectangular cavity with heat generation having non-uniform temperature profile in the wall. The aim of the present investigation is to study on the magnetohydrodynamic natural convection flow in a rectangular cavity filled with heat generating fluid having linearly heated bottom wall.

\section{Geometry and equations of motion}

A rectangular cavity filled with viscous incompressible fluid is shown in the Fig. 1. The cavity dimensions are defined by $L$ for width and $\mathrm{H}$ for height. The cavity is isothermally heated from the left vertical wall with a uniform constant temperature $T_{h}$ and the right vertical with temperature $T_{c}\left(T_{h}>T_{c}\right)$. The bottom horizontal wall is linearly heated with $T_{h}-\left(T_{h}-T_{c}\right) \frac{x}{L}$ while the remaining wall is considered perfectly insulated.

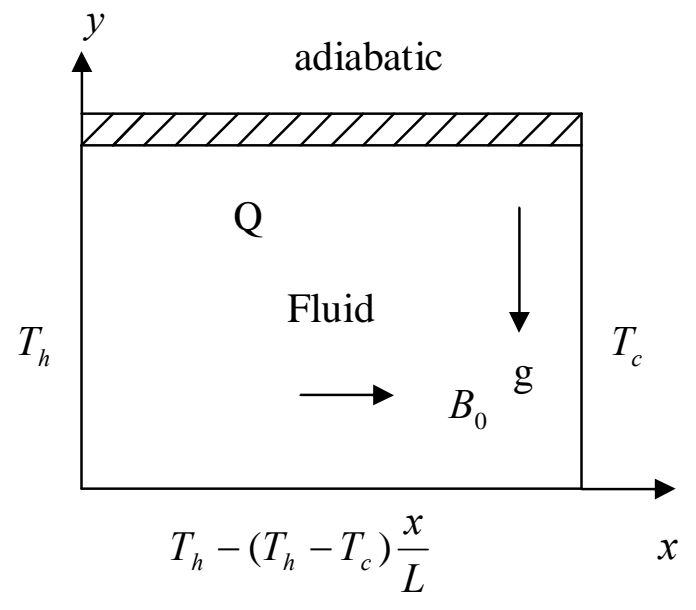

Fig. 1 The flow configuration and coordinate system 
The governing equations of the problem under consideration are based on the balance laws of mass, linear momentum and energy modified to account for the presence of thermal buoyancy, magnetic field and the heat generation or absorption effects. Nondimensional quantities used for making the governing equations into dimensionless form are stated as follows:

$$
X=\frac{x}{L}, Y=\frac{y}{L}, U=\frac{u L}{\alpha}, V=\frac{v L}{\alpha}, P=\frac{p L^{2}}{\rho \alpha^{2}}, \theta=\frac{T-T_{c}}{T_{h}-T_{c}}
$$

where $X$ and $Y$ are the coordinates varying along horizontal and vertical directions, respectively, $U$ and $V$ are, the velocity components in the $X$ and $Y$ directions respectively, $\theta$ is the dimensionless temperature and $P$ is the dimensionless pressure. After substitution of the dimensionless variables into the governing equations we get the following dimensionless equations:

$$
\begin{aligned}
& \frac{\partial U}{\partial X}+\frac{\partial V}{\partial Y}=0 \\
& U \frac{\partial U}{\partial X}+V \frac{\partial U}{\partial Y}=-\frac{\partial P}{\partial X}+\operatorname{Pr}\left(\frac{\partial^{2} U}{\partial X^{2}}+\frac{\partial^{2} U}{\partial Y^{2}}\right) \\
& U \frac{\partial V}{\partial X}+V \frac{\partial V}{\partial Y}=-\frac{\partial P}{\partial Y}+\operatorname{Pr}\left(\frac{\partial^{2} V}{\partial X^{2}}+\frac{\partial^{2} V}{\partial Y^{2}}\right)+R a_{E} \operatorname{Pr} \theta-H a^{2} \operatorname{Pr} V \\
& U \frac{\partial \theta}{\partial X}+V \frac{\partial \theta}{\partial Y}=-\frac{\partial P}{\partial X}+\left(\frac{\partial^{2} \theta}{\partial X^{2}}+\frac{\partial^{2} \theta}{\partial Y^{2}}\right)+\frac{R a_{E}}{R a_{I}}
\end{aligned}
$$

The dimensionless parameters appearing in the Eqs. (1)-(4) are the external Rayleigh number $R a_{E}$, internal Rayleigh number $R a_{I}$, Prandtl number $\mathrm{Pr}$, Hartmann number $\mathrm{Ha}$, They are defined as follows:

$$
R a_{E}=\frac{g \beta\left(T_{h}-T_{c}\right) L^{3}}{v \alpha}, R a_{I}=\frac{g \beta Q L^{5}}{v \alpha k}, \operatorname{Pr}=\frac{v}{\alpha} \text { and } H a^{2}=\frac{\sigma B_{0}{ }^{2} L^{2}}{\mu}
$$

where $g$ is the gravitational constant, $\beta$ is the volumetric coefficient of thermal expansion, $\mathrm{Q}$ is the rate of internal heat generation per unit volume, $B_{0}$ is the magnetic induction, $\alpha$ is the thermal diffusivity and $v$ kinematic viscosity of the fluid.

The dimensionless boundary conditions are:

$$
\begin{aligned}
& \mathrm{U}(\mathrm{X}, 0)=\mathrm{U}(\mathrm{X}, \mathrm{A})=\mathrm{U}(0, \mathrm{Y})=\mathrm{U}(1, \mathrm{Y})=0 \\
& \mathrm{~V}(\mathrm{X}, 0)=\mathrm{V}(\mathrm{X}, \mathrm{A})=\mathrm{V}(0, \mathrm{Y})=\mathrm{V}(1, \mathrm{Y})=0 \\
& \theta(\mathrm{X} .0)=1-\mathrm{X} \quad \theta(0, \mathrm{Y})=1, \theta(1, \mathrm{Y})=0 \\
& \frac{\partial \theta}{\partial Y}(X, A)=0
\end{aligned}
$$


where $A=H / L$ is the aspect ratio of the rectangular cavity which is taken as 0 .75. The heat transfer coefficient in terms of local Nusselt number is defined by $N u=-\frac{\partial \theta}{\partial n}$ where $\mathrm{n}$ denotes the normal direction on a plane. The average Nusselt numbers at the bottom and side walls are computed as $\overline{N u_{b}}=\int_{0}^{1} N u_{b} d X$, $\overline{N u_{s}}=\frac{1}{A} \int_{0}^{A} N u_{s} d Y$

\section{Numerical method and choice of parameter}

The governing Eqs.(1)-(4) along with the boundary conditions are solved numerically, employing finite element method. The six nodded triangular element is used in this work for the development of the finite element equations. All six nodes are associated with velocities as well as temperature; only the corner nodes are associated with pressure. This means that a lower order polynomial is chosen for pressure.

The velocity components, temperature distribution and pressure distribution according to their highest derivative orders in the differential equations is considered as

$U(X, Y)=N_{\beta} U_{\beta}$

$V(X, Y)=N_{\beta} V_{\beta}$

$T(X, Y)=N_{\beta} T_{\beta}$

$P(X, Y)=H_{\lambda} P_{\lambda}$

where $N_{\alpha}(\alpha=1,2$, é é ,6) are the element shape functions for the velocity components and the temperature, and $H_{\lambda}(\lambda=1,2,3)$ are the element shape functions for the pressure.

To derive the finite element equations, the method of weighted residuals is applied to the Eqs. (1)ï (4) according to Zienkiewicz [13] as

$$
\begin{aligned}
& \int_{A} H_{\lambda}\left(\frac{\partial U}{\partial X}+\frac{\partial V}{\partial Y}\right) d A=0 \\
& \int_{A} N_{\alpha}\left(U \frac{\partial U}{\partial X}+V \frac{\partial U}{\partial Y}\right) d A=-\int_{A} N_{\alpha}\left(\frac{\partial P}{\partial X}\right) d A+\operatorname{Pr} \int_{A} N_{\alpha}\left(\frac{\partial^{2} U}{\partial X^{2}}+\frac{\partial^{2} V}{\partial Y^{2}}\right) d A
\end{aligned}
$$




$$
\begin{aligned}
& \int_{A} N_{\alpha}\left(U \frac{\partial V}{\partial X}+V \frac{\partial V}{\partial Y}\right) d A=-\int_{A} N_{\alpha}\left(\frac{\partial P}{\partial Y}\right) d A+\operatorname{Pr} \int_{A} N_{\alpha}\left(\frac{\partial^{2} U}{\partial X^{2}}+\frac{\partial^{2} V}{\partial Y^{2}}\right) d A \\
&+R a \operatorname{Pr} \int_{A} N_{\alpha} \theta d A-H a^{2} \int_{A} N_{\alpha} V d A \\
& \int_{A} N_{\alpha}\left(U \frac{\partial \theta}{\partial X}+V \frac{\partial \theta}{\partial Y}\right) d A=\int_{A} N_{\alpha}\left(\frac{\partial^{2} U}{\partial X^{2}}+\frac{\partial^{2} V}{\partial Y^{2}}\right) d A+\frac{R a_{E}}{R a_{I}} \int_{A} N_{\alpha} d A
\end{aligned}
$$

where $A$ is the element area.

Gauss divergence theorem is then applied to Eqs. (10)-(12) to generate the boundary integral terms associated with the surface tractions and heat flux. Then Eqs. (10)-(12) become,

$$
\begin{gathered}
\int_{A} N_{\alpha}\left(U \frac{\partial U}{\partial X}+V \frac{\partial U}{\partial Y}\right) d A+\int_{A} N_{\alpha}\left(\frac{\partial P}{\partial X}\right) d A+\operatorname{Pr} \int_{A}\left(\frac{\partial N_{\alpha}}{\partial X} \frac{\partial U}{\partial X}+\frac{\partial N_{\alpha}}{\partial Y} \frac{\partial U}{\partial Y}\right) d A \\
=\int_{S_{0}} N_{\alpha} S_{x} d S_{0} \\
\int_{A} N_{\alpha}\left(U \frac{\partial V}{\partial X}+V \frac{\partial V}{\partial Y}\right) d A+\int_{A} N_{\alpha}\left(\frac{\partial P}{\partial Y}\right) d A+\operatorname{Pr} \int_{A}\left(\frac{\partial N_{\alpha}}{\partial X} \frac{\partial V}{\partial X}+\frac{\partial N_{\alpha}}{\partial Y} \frac{\partial V}{\partial Y}\right) d A+ \\
\operatorname{Ra} \operatorname{Pr} \int_{A} N_{\alpha} \theta d A-H a^{2} \int_{A} N_{\alpha} V d A=\int_{S_{0}} N_{\alpha} S_{y} d S_{0} \\
\int_{A} N_{\alpha}\left(U \frac{\partial T}{\partial X}+V \frac{\partial T}{\partial Y}\right) d A+\int_{A}\left(\frac{\partial N_{\alpha}}{\partial X} \frac{\partial T}{\partial X}+\frac{\partial N_{\alpha}}{\partial Y} \frac{\partial T}{\partial Y}\right) d A+\frac{R a_{E}}{R a_{I}} \int_{A} N_{\alpha} d A \\
=\int_{S_{0}} N_{\alpha} q_{w} d S_{w}
\end{gathered}
$$

Here Eqs.(13)-(14) specifies surface tractions $\left(S_{x}, S_{y}\right)$ along outflow boundary $S_{0}$ and Eq.(15) specifies heat flux $\left(q_{w}\right)$ that flows into or out from domain along wall boundary $S_{w}$.

Substituting the element velocity component distributions, the temperature distribution, and the pressure distribution in Eq. (9)and Eqs.(13)-(15) the finite element equations can be written in the form,

$$
\begin{aligned}
& K_{\alpha \beta^{x}} U_{\beta}+K_{\alpha \beta^{y}} V_{\beta}=0 \\
& K_{\alpha \beta \gamma^{x}} U_{\beta} U_{\gamma}+K_{\alpha \beta \gamma^{y}} V_{\beta} U_{\gamma}+M_{\alpha \mu^{x}} P_{\mu}+\operatorname{Pr}\left(S_{\alpha \beta^{x x}}+S_{\alpha \beta^{y y}}\right) U_{\beta}=Q_{\alpha u} \\
& K_{\alpha \beta \gamma^{x}} U_{\beta} V_{\gamma}+K_{\alpha \beta \gamma^{y}} V_{\beta} V_{\gamma}+M_{\alpha \mu^{y}} P_{\mu}+\operatorname{Pr}\left(S_{\alpha \beta^{x x}}+S_{\alpha \beta^{y y}}\right) V_{\beta} \\
& \quad+\operatorname{Ra} \operatorname{Pr} K_{\alpha \beta} \theta_{\beta}-H a^{2} \operatorname{Pr} K_{\alpha \beta} V_{\beta}=Q_{\alpha} v
\end{aligned}
$$




$$
K_{\alpha \beta \gamma^{\gamma}} U_{\beta} \theta_{\gamma}+K_{\alpha \beta \gamma^{y}} V_{\beta} \theta_{\gamma}+M_{\alpha \mu^{Y}} P_{\mu}+\left(S_{\alpha \beta^{x x}}+S_{\alpha \beta^{y y}}\right) \theta_{\beta}+\frac{R a_{E}}{R a_{I}} K_{\alpha \beta}=Q_{\alpha} \theta
$$

where the coefficients are in the form of the integrals over the element area and along the element edges $S_{0}$ and $S_{w}$ as

$$
\begin{aligned}
& K_{\alpha \beta \gamma^{x}}=\int_{A} N_{\alpha} N_{\beta} N_{\gamma, x} d A \\
& S_{\alpha \beta^{x x}}=\int_{A} N_{\alpha, x} N_{\beta, x} d A \\
& M_{\alpha \mu^{x}}=\int_{A} H_{\alpha} H_{\mu, x} d A \\
& Q_{\alpha^{u}}=\int_{S_{0}} N_{\alpha} S_{x} d S_{0} \\
& Q_{\alpha^{\theta}}=\int_{S_{w}} N_{\alpha} q_{1 w} d S_{w}
\end{aligned}
$$

The integration involved in each term is evaluated numerically by using Gauss-Legendre quadrature method.

The finite element Eqs. (17)-(19), are nonlinear. These nonlinear algebraic equations are solved by applying the Newton-Raphson iteration technique by first writing the unbalanced values from the set of the finite element Eqs. (16)-(19) as,

$$
\begin{aligned}
& F_{\alpha} p=K_{\alpha \beta^{x}} U_{\beta}+K_{\alpha \beta^{y}} V_{\beta} \\
& F_{\alpha^{u}}=K_{\alpha \beta \gamma^{x}} U_{\beta} U_{\gamma}+K_{\alpha \beta \gamma^{y}} V_{\beta} U_{\gamma}+M_{\alpha \mu^{x}} P_{\mu}+\operatorname{Pr}\left(S_{\alpha \beta^{x x}}+S_{\alpha \beta^{y y}}\right) U_{\beta}-Q_{\alpha^{u}} \\
& F_{\alpha^{v}}=K_{\alpha \beta \gamma^{x}} U_{\beta} V_{\gamma}+K_{\alpha \beta \gamma^{y}} V_{\beta} V_{\gamma}+M_{\alpha \mu^{Y}} P_{\mu}+\operatorname{Pr}\left(S_{\alpha \beta^{x x}}+S_{\alpha \beta^{y y}}\right) V_{\beta} \\
& +R a \operatorname{Pr} K_{\alpha \beta} \theta_{\beta}-H a^{2} \operatorname{Pr} K_{\alpha \beta} V_{\beta}-Q_{\alpha} v \\
& F_{\alpha^{\theta}}=K_{\alpha \beta \gamma^{x}} U_{\beta} \theta_{\gamma}+K_{\alpha \beta \gamma^{y}} V_{\beta} \theta_{\gamma}+M_{\alpha \mu^{Y}} P_{\mu}+\left(S_{\alpha \beta^{x x}}+S_{\alpha \beta^{y y}}\right) \theta_{\beta} \\
& +\frac{R a_{E}}{R a_{I}} K_{\alpha \beta}-Q_{\alpha} \theta
\end{aligned}
$$

This leads to a set of algebraic equations with the incremental unknowns of the element nodal velocity components, temperatures, and pressures in the form,

$$
\left[\begin{array}{cccc}
K_{u u} & K_{u v} & K_{u \theta} & K_{u p} \\
K_{v u} & K_{v v} & K_{v \theta} & K_{v p} \\
K_{\theta u} & K_{\theta v} & K_{\theta \theta} & 0 \\
K_{p u} & K_{p v} & 0 & 0
\end{array}\right]\left\{\begin{array}{c}
\Delta u \\
\Delta v \\
\Delta \theta \\
\Delta p
\end{array}\right\}=-\left\{\begin{array}{c}
F_{\alpha^{U}} \\
F_{\alpha^{V}} \\
F_{\alpha^{\theta}} \\
F_{\alpha^{U}}
\end{array}\right\}
$$

where 
$K_{u u}=K_{\alpha \beta \gamma^{x}} U_{\beta}+K_{\alpha \beta \gamma^{x}} U_{\gamma}+K_{\alpha \beta \gamma^{y}} V_{\beta}++\operatorname{Pr}\left(S_{\alpha \beta^{x x}}+S_{\alpha \beta^{y y}}\right)$

An assessment of the accuracy of the numerical simulation procedure is of course of fundamental importance. The discretization process involves a certain amount of error, which can be systematically reduced by a series of grid refinements. To this end, five types of grid densities have been chosen to check for the self-consistency of the present study. The grids chosen are: (a) 22342 nodes, 3453 elements, (b) 28455 nodes, 4421 elements (c) 35796 nodes, 5589 elements (d) 41124 nodes, 6424 elements (e) 47212 nodes, 7387 elements. Type (c) mesh density was found to give sufficient accuracy with modest computational time, and hence selected for the simulation study.

To ensure convergence of solutions the following criteria is applied to all dependent variables over the solution domain

$$
\Sigma\left|\Phi_{i, j}{ }^{n}-\Phi_{i, j}{ }^{n-1}\right| \leq E R M A X
$$

where $\Phi$ represents the dependent variables $\mathrm{U}, \mathrm{V}, \mathrm{P}$ and $\mathrm{T}$; the indexes $\mathrm{i}, \mathrm{j}$ refers to space coordinates and the index $\mathrm{n}$ is the current iteration.. The value of ERMAX is chosen as $10^{-5}$.

The parameter used in this study are Hartmann number. external Rayleigh number and internal Rayleigh number. The fundamental concept behind MHD is that magnetic fields can induce currents in a moving conductive fluid, which in turn creates forces on the fluid and also changes the magnetic field itself. Hartmann number is the ratio of electromagnetic force to the viscous force. The extent of the effect of magnetic field on the flow is determined by the magnitude of the Hartmann number.

The external Rayleigh number is the ratio of buoyancy and viscosity forces times the ratio of momentum and thermal diffusivities. The extent of the effect of lateral heat on the flow is determined by the magnitude of the external Rayleigh number. The effect of internal heat generation on the flow is determined by the magnitude of the internal Rayleigh number.

The present code was exercised on the work of Sathiyamoorty et al.[12] to check its validity. We recall here some results obtained by our code in comparison to those reported in Sathiyamoorty et al.[12] for $\mathrm{Ra}_{\mathrm{E}}=10^{3}, 10^{4}, 10^{5}$. Table 1 . shows that the present results have a good agreement with those obtained by Sathiyamoorty et al.[12].

Table 1. Nusselt Number comparison for $\operatorname{Pr}=0.71$

\begin{tabular}{|l|l|l|l|}
\hline $\mathrm{Ra}_{\mathrm{E}}$ & Present work & Sathiyamoorty et al. & Error $(\%)$ \\
\hline $10^{3}$ & 3.7196 & 3.7294 & 0.26 \\
\hline $10^{4}$ & 4.7520 & 4.7753 & 0.34 \\
\hline $10^{5}$ & 6.8042 & 6.8272 & 0.33 \\
\hline
\end{tabular}




\section{Results and Discussion}

Numerical results are presented in order to determine the effects of the presence of magnetic field, volumetric heat generation and lateral heat difference on the natural convection flow of an electrically conducting fluid in a rectangular cavity. It is seen that there are three governing parameters in this problem: the Hartmann number $H a$, the internal Rayleigh number $R a_{I}$, the external Rayleigh number $R a_{E}$.

Values of the Hartmann number $\mathrm{Ha}$ range from 0.0 to 50.0, the external Rayleigh number $R a_{E}$ from $10^{2}$ to $10^{5}$ and the internal Rayleigh number $R a_{I}$ from 00 to $10^{5}$ while $P r=0.71$ is kept fixed. All four boundaries are considered to be rigid $(\mathrm{u}=\mathrm{v}=0)$. The top wall is assumed to be adiabatic $(\partial \theta / \partial Y=0)$ and the bottom wall is linearly heated with temperature $T_{h}-\left(T_{h}-T_{c}\right) \frac{x}{L}$. The left wall is maintained at temperature $T_{h}$ while the right wall is held at temperature $T_{c}\left(T_{h}>T_{c}\right)$.

\subsection{Flow and temperature fields}

\subsubsection{Effects of Internal Rayleigh numbers}

The effect of internal heat generation on the flow field has been considered. The resulting flow and temperature distribution has been depicted in Figs. 2-5 where the top row gives the streamlines for increasing values of the internal Rayleigh numbers $R a_{I}=00,10^{3}, 10^{4}$ and $10^{5}$ while the external Rayleigh number $R a_{E}=10^{3}$, the Hartmann number $H a=20$ and the Prandtl number $P r=0.71$ are kept fixed with linearly heated bottom wall where the top wall is well insulated. The left and right vertical wall are maintained at hot and cold temperature respectively.

In these figures we see that without heat generation there is only one cell called primary cell . With the effect of the internal Rayleigh number a secondary cell has been developed in top left corner of the cavity. The increasing rate of heat within the cavity due to the increase of the internal Rayleigh Number leads to increase the flow rate in the secondary cell as well as increase in its size until it occupies half of the cavity. This effect of internal heat generation on the flow field is reasonable since internal heat generation assists the buoyancy forces by accelerating the fluid flow.

On the other hand the fluid temperature increases significantly due to effect of the internal Rayleigh number which is shown in the isotherms of Figs. 2-5 (bottom). It is clearly seen that owing to the increase of the internal Rayleigh Number the fluid temperature exceeds the surface temperature.

\subsubsection{Effects of External Rayleigh numbers}

Figs. 6-9 illustrate the stream functions and isotherm contours for various values of $R a_{E}$ $=10^{2}, 10^{3}, 10^{4}$ and $10^{5}$ where $H a=20, R a_{I}=10^{4}$ and $P r=0.71$ is kept fixed with linearly heated bottom wall where the top wall is well insulated the left and right vertical walls are maintained at hot and cold temperature respectively. For the lower external Rayleigh number two convective cells dominate the flow, however with the increase of the external Rayleigh number, primary cell becomes larger and occupies whole domain when $R a_{E}=$ $10^{4}$. This behaviour is almost opposite to the effect of the internal Rayleigh number from 

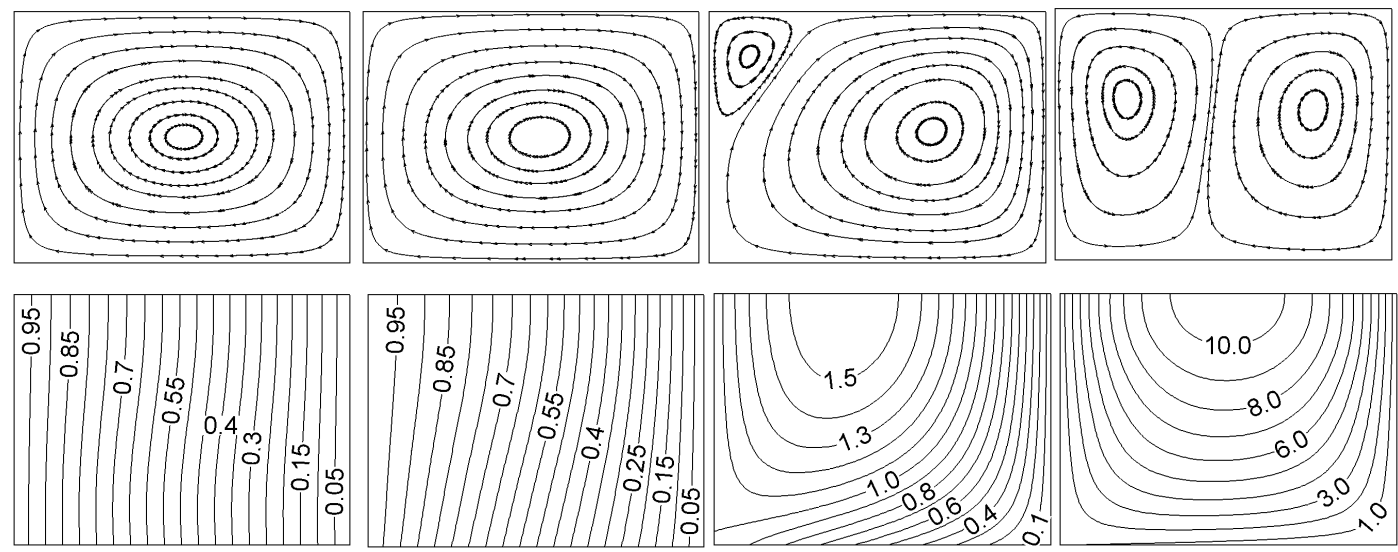

Fig. 2. Contour

Fig. 3. Contour

plots for $R a_{E}=10^{3}$, plots for $R a_{E}=10^{3}$

$R a_{I}=00, H a=20 \quad R a_{I}=10^{3}, H a=20$

streamline(top); $\quad$ streamline(top);

isotherm(bottom) isotherm(bottom)
Fig. 4. Contour

plots for $R a_{E}=10^{3}$

$R a_{I}=10^{4}, H a=20$

streamline(top);

isotherm(bottom)
Fig. 5. Contour plots for $R a_{E}=10^{3}$

$R a_{I}=10^{5}, H a=20$ streamline(top); isotherm(bottom)

lower to higher, for a fixed external Rayleigh number. As we discussed earlier that the internal Rayleigh number accelerates the flow and eventually it has an influence on buoyancy, however, for a fixed internal Rayleigh number when external Rayleigh number increases; the buoyancy effect accelerates and dominates the flows although
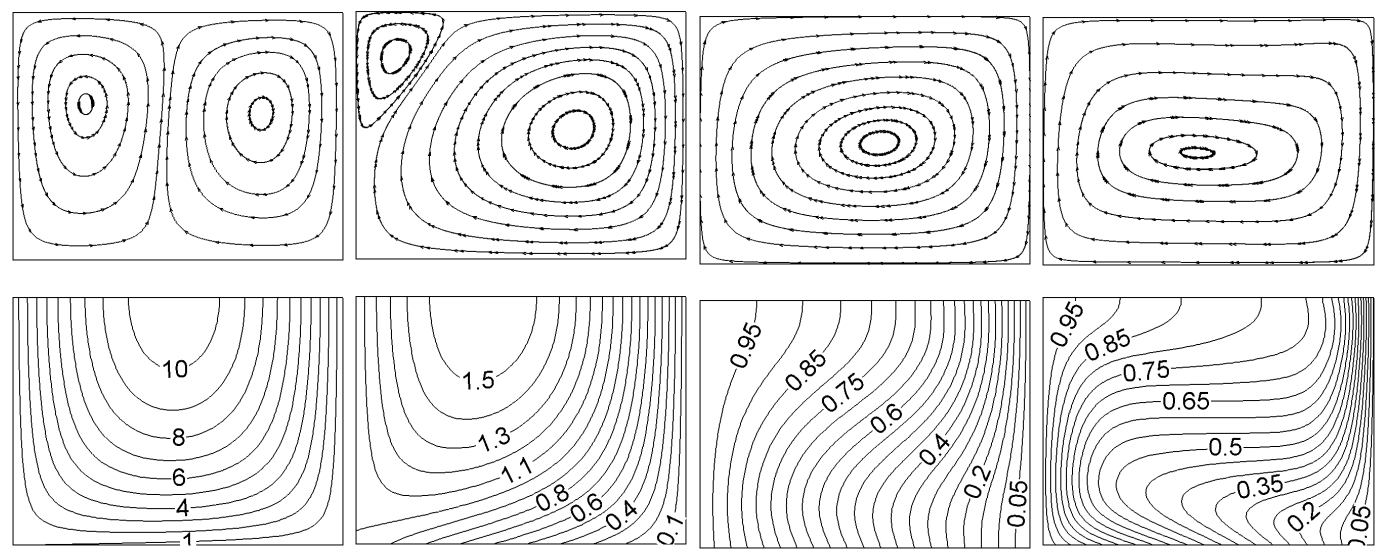

Fig. 6. Contour

Fig. 7. Contour

plots for $R a_{E}=10^{2}$, plots for $R a_{E}=10^{3}$

$R a_{I}=10^{4}, H a=20 \quad R a_{I}=10^{4}, H a=20$

streamline(top); $\quad$ streamline(top);

isotherm(bottom) isotherm(bottom)
Fig. 8. Contour

plots for $R a_{E}=10^{4}$

$R a_{I}=10^{4}, H a=20$

streamline(top);

isotherm(bottom)
Fig. 9. Contour

plots for $R a_{E}=10^{5}$

$R a_{I}=10^{4}, H a=20$

streamline(top);

isotherm(bottom) 
there is a weak internal Rayleigh number influence on the flow field. The same phenomenon has been seen on the temperature contours as shown in Figs. 6-9(bottom). Initially when the external Rayleigh number is small, the internal fluid temperature is higher, but it decreases with the increase of $\mathrm{Ra}_{\mathrm{E}}$. As $\mathrm{Ra}_{\mathrm{E}}$ increases from $10^{3}$ to $10^{5}$ the flow rate increases. At $\mathrm{Ra}_{\mathrm{E}}=10^{4}$ the circulation are stronger and consequently the temperature contours are pushed towards the right corner of the cavity.

\subsubsection{Effects of Hartmann numbers}

Figs. 10-13 depict the influence of Hartmann number $\mathrm{Ha}$ on the flow and temperature fields for various values of $H a=00,10,20,50$ where external Rayleigh number $R a_{E}=10^{3}$, internal Rayleigh number $R a_{I}=10^{4}$ and $P r=0.71$ is kept fixed . Because of linearly heated bottom wall and uniformly heated left vertical wall fluid rises up from the left portion of the bottom wall and flow down along the right wall forming a roll with clock wise rotation inside the cavity as shown in the Fig. 2(top) with $R a_{E}=10^{3}$ and $R a_{1}=00$.

But here in the Fig. 10(top) two cells are formed due to the fact that the internal Rayleigh number is greater than the external Rayleigh number as shown in the Fig. 4.(top) with $R a_{E}=10^{3}$ and $R a_{I}=10^{4}$. The left cell revolves anticlockwise because of greater internal Rayleigh number and the right cell revolves clockwise as expected.
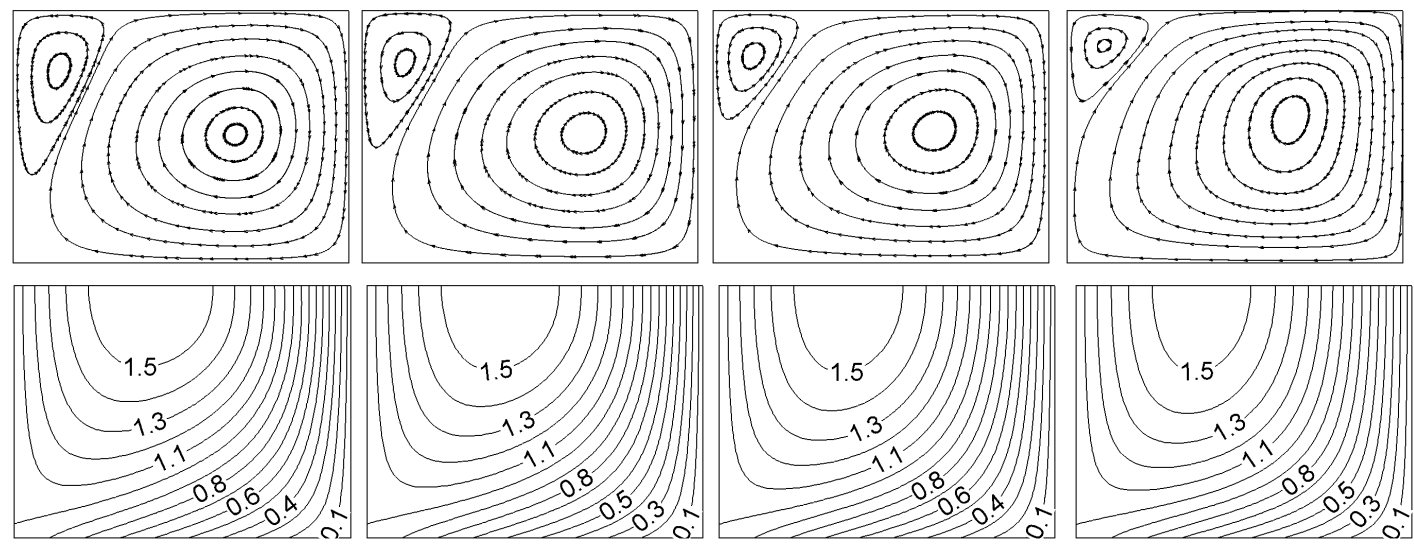

Fig. 10. Contour Fig. 11. Contour

plots for $R a_{E}=10^{3}$, plots for $R a_{E}=10^{3}$ $R a_{I}=10^{4}, H a=00 \quad R a_{I}=10^{4}, H a=10$

streamline(top); streamline(top);

isotherm(bottom) isotherm(bottom)
Fig. 12. Contour

plots for $R a_{E}=10^{3}$

$R a_{I}=10^{4}, H a=20$

streamline(top);

isotherm(bottom)
Fig. 13. Contour

plots for $R a_{E}=10^{3}$

$R a_{I}=10^{4}, H a=50$

streamline(top);

isotherm(bottom)

From the figures as shown in the top rows, it can be seen that intensities of the flow decrease owing to increase in the magnetic field. Because of the decrease of the intensity of the flow the left cell is becoming smaller. This is expected since presence of magnetic field usually retards the velocity field. 
The corresponding effect of the increasing magnetic field on the isotherms may be viewed from the Figs. 10-13(bottom). It is seen that the isotherms become more curved due to the increase of the magnetic field strength, which is expected; since the magnetic field resists the flow.

\subsection{Heat transfer rates}

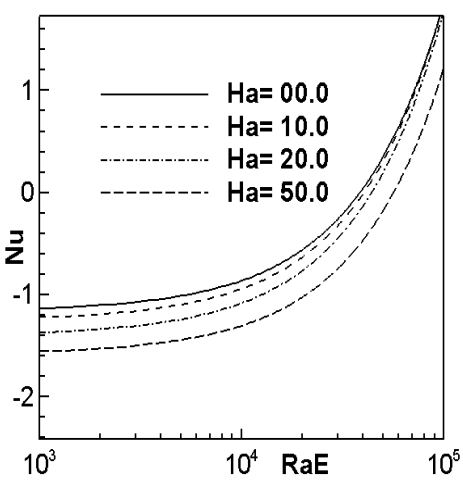

(a)

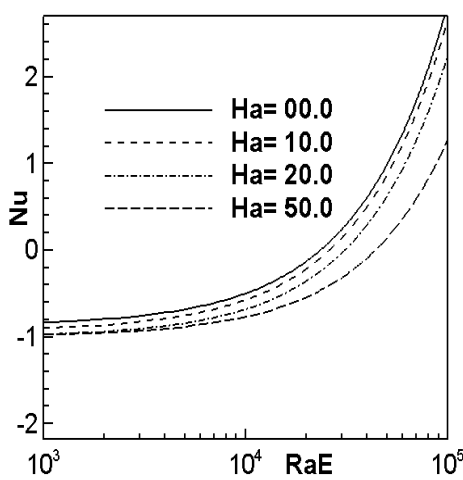

(b)

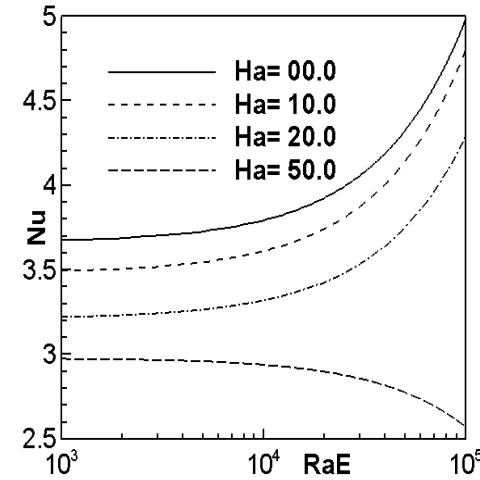

(c)

Fig.14 Variation of average Nusselt number with external Rayleigh number for (a) linearly heated bottom wall, (b) heated left wall and (c) cooled right wall for Hartmann number $H a=0.0,10,20$ and 50 .

The overall effects of $R a_{E}$ and $H a$ on the average Nusselt number for linearly heated bottom wall, heated left wall and cooled right wall for $\operatorname{Pr}=0.71$, internal Rayleigh number $R a_{I}=10^{4}$ and Hartmann numbers $H a=0.0,10,20$ and 50. are displayed in Figs. 14(a), (b) and (c) via average Nusselt number vs external Rayleigh number plot.

Initially at $R a_{E}=10^{3}$ the heat transfer on the bottom and left wall is negative. From Fig. 14(a) and (b) it is observed that the average Nusselt numbers smoothly increase due to dominant heat conduction mode and becomes positive when the external Rayleigh number $R a_{E}$ is $3 \times 10^{4}$ for the bottom wall and $2 \times 10^{4}$ for the left wall. Then the Nusselt number increases rapidly as Rayleigh number increases further.

From the Fig. 14(c) it is seen that at $R a_{E}=10^{3}$ the heat transfer on the right wall is positive and increases smoothly for $R a_{E}$ from $10^{3}$ to $10^{5}$ in case of $H a=0.0,10$ and 20 but for $R a_{E}$ from $10^{4}$ to $10^{5}$ Nusselt numbers decreases for $H a=50$.

The heat transfer characteristics of the present study are of technological importance, mainly because of the presence of the magnetic field. The increase of the Hartmann number causes reduction of the heat transfer rates from the cavity surfaces. This phenomenon is related to the damping effect of the increasing magnetic field which results in the domination of conduction over convection heat transfer. 


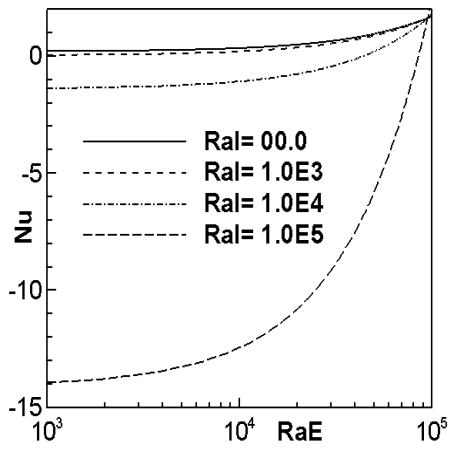

(a)

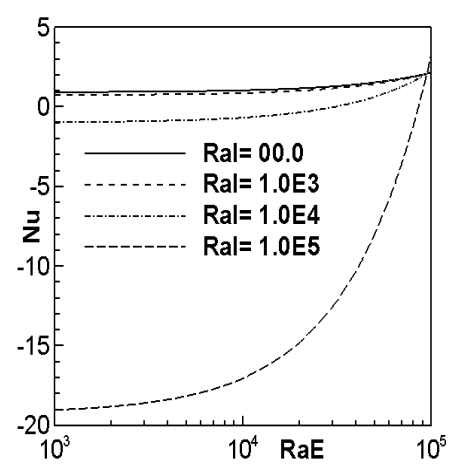

(b)

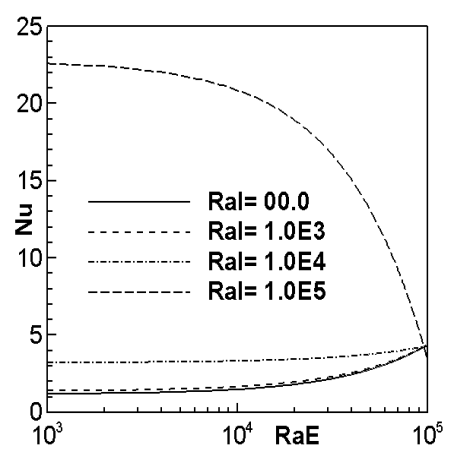

(c)

Fig.15 Variation of average Nusselt number with external Rayleigh number for (a) linearly heated bottom wall, (b) heated left wall and (c) cooled right wall for internal Rayleigh number $R a_{I}=0.0,10^{3}, 10^{4}$ and $10^{5}$

The overall effects of $R a_{E}$ and $R a_{I}$ on the average Nusselt number for linearly heated bottom wall, heated left wall and cooled right wall for $\operatorname{Pr}=0.71$, external Rayleigh number for $R a_{E}=10^{3}$ and internal Rayleigh number $R a_{I}=0.0,10^{3}, 10^{4}$ and $10^{5}$ are displayed in fig. 15(a), (b) and (c) via average Nusselt number vs external Rayleigh number plot.

It is seen that the average Nusselt Number is negative for both the linearly heated bottom wall and heated left wall. But it is positive for the cooled right wall. In all the cases heat transfer is very high for $R a_{I}=10^{5}$ in all the walls. But it is very low in all other cases.

\section{Conclusion}

The influence of linearly heated bottom walls with uniformly heated left wall on flow and heat transfer characteristic due to natural convection within a rectangular enclosure having heat generating and electrically conducting fluid has been studied in the present investigation. An analysis for the distribution of streamlines, isotherms, average Nusselt numbers was performed to study the effect of dimensionless parameters. The investigation was carried out for a number of dimensionless groups namely the external Rayleigh number $R a_{E}$, internal Rayleigh number $R a_{I}$ and Hartmann number $\mathrm{Ha}$. From the present investigation the following conclusion may be drawn:

- Increase in the value of external Rayleigh numbers leads to increase the size of the primary cell until it occupies the whole cavity space. Also the circulation gets stronger and consequently the temperature contours are pushed towards the right corner of the cavity. Rate of heat transfer from the heated left wall and bottom wall increases due to the increase of external Rayleigh number.

- The temperature of the fluid in the cavity increases due to the increase of the internal Rayleigh number, consequently the rate of heat transfer from the left wall and bottom wall decreases. Increase in the value of internal Rayleigh number leads to develop a secondary cell on the left corner of the cavity and increases its size until it occupies almost half of the cavity. 
- The significant effect of the magnetic field is observed in the heat transfer mechanisms and flow characteristics inside the cavity. Strong suppression of the convective current can be obtained by applying strong magnetic field. This is why; reductions in the average Nusselt number $N u$ were produced as the strength of the applied magnetic field were increased.

\section{REFERENCES}

[1] Chamkha, A. J., ñHydromagnetic combined convection flow in a vertical lid-driven cavity with internal heat generation or absorptionò, Numer. Heat Transfer, Part A, Vol. 41, pp. 529-546, 2002.

[2] Garandet, J. P., Alboussiere, T., and Moreau, R., ñBuoyancy driven convection in a rectangular enclosure with a transverse magnetic fieldò, International Journal of Heat and Mass Transfer, Vol. 35, pp. 741-748, 1992.

[3] Hossain M. A., Hafiz M.Z and Rees D.A.S., ñ̃uoyancy and thermo capillary driven convection flow of an electrically conducting fluid in an enclosure with heat generation.ò International Journal of Thermal Sciences. Vol. 44, pp. 676ї 684, 2005

[4] Kahveci, K and Oztuna, S. ñMHD natural convection flow and heat transfer in a laterally heated partitioned enclosureò, European Journal of Mechanics, Vol. 28, pp 744-752, 2009.

[5] Kandaswamy, P., Sundari, S.M and Nithyadevi, N. ñMagneto convection in an enclosure with partially active vertical wallsò, International Journal of Heat and Mass Transfer, Vol. 51, pp. 1946-1954, 2008.

[6] Mehmet, C. E. and Elif, B., ñNatural convection flow under a magnetic field in an inclined rectangular enclosure heated and cooled on adjacent walls.ò, Fluid Dynamics Research, Vol. 38, pp. 564ї 590, 2006.

[7] Nithyadevi, N., Kandaswamy, P. and Sundari, S.M. ñMagnetoconvection in a square cavity with partially active vertical walls: Time periodic boundary conditionò International Journal of Heat and Mass Transfer, Vol. 52 pp. 1945-1953, 2009

[8] Oreper, G. M., Szekely, J., ñThe effect of an externally imposed magnetic field on buoyancy driven flow in a rectangular cavityò, J. of Crystal Growth, Vol. 64, pp. 505-515, 1983.

[9] Ozoe, H., and Maruo, M., ñMagnetic and gravitational natural convection of melted silicontwo dimensional numerical computations for the rate of heat transferò, JSME, Vol. 30, pp. 774-784, 1987.

[10] Rudraiah, N., Barron, R. M., Venkatachalappa, M., and Subbaraya, C. K., r̃Effect of magnetic field on free convection in a rectangular enclosureò, Int. J. Engg. Sci., Vol. 33, pp. 1075-1084, 1995.

[11] Sarris, I. E., Kakarantzas, S. C., Grecos, A. P., and Vlachos, N. S., ñMHD natural convection in a laterally and volumetrically heated square cavityò, Int. J. of Heat and Mass Transfer, Vol. 48, pp. 3443-3453, 2005.

[12] Sathiyamoorty, M., Tanmay Basak, Roy, S. and Pop, I. ñSteady Natural convection flow in a square cavity with linearly heated side walls.ò International Journal of Heat and Mass Transfer, Vol. 50 pp. 766-775, 2007.

[13] Zienkiewicz, O. C. and Taylor, R. L., ñThe finite element methodò, Fourth Ed., McGraw-Hill, 1991. 